\title{
Gene Expression Profiling of Xeroderma Pigmentosum
}

\author{
Nikola A. Bowden ${ }^{1,2}$, Paul A. Tooney ${ }^{1,2}$, Rodney J. Scott ${ }^{1-3}$ \\ 1Hunter Medical Research Institute, John Hunter Hospital, Lookout Rd, New Lambton Heights, NSW, 2305, Australia; 2School of Biomedical Sciences, University of \\ Newcastle, Callaghan 2308, Australia; 3Division of Genetics, Hunter Area Pathology Service, John Hunter Hospital, Lookout Rd, New Lambton Heights 2305, Australia
}

Key words: Xeroderma Pigmentosum, Nucleotide excision repair, gene expresion profiling, UV-light and neurological symptoms

Corresponding author: Nikola A. Bowden, Hunter Medical Research Institute, Level 3 John Hunter Hospital, Lookout Rd, New Lambton Heights NSW 2305, Australia, tel.: +61 24985 5893, fax: +61 249214196 , e-mail: nikola.bowden@newcastle.edu.au; Paul A. Tooney: paul.tooney@newcastle.edu.au; Rodney J. Scott: rodney.scott@newcastle.edu.au

Submitted: 3 May 2006

Accepted: 20 May 2006

\begin{abstract}
Xeroderma pigmentosum (XP) is a rare recessive disorder that is characterized by extreme sensitivity to UV light. UV light exposure results in the formation of DNA damage such as cyclobutane dimers and (6-4) photoproducts. Nucleotide excision repair (NER) orchestrates the removal of cyclobutane dimers and (6-4) photoproducts as well as some forms of bulky chemical DNA adducts. The disease XP is comprised of 7 complementation groups (XP-A to XP-G), which represent functional deficiencies in seven different genes, all of which are believed to be involved in NER. The main clinical feature of XP is various forms of skin cancers; however, neurological degeneration is present in XPA, XPB, XPD and XPG complementation groups. The relationship between NER and other types of DNA repair processes is now becoming evident but the exact relationships between the different complementation groups remains to be precisely determined.

Using gene expression analysis we have identified similarities and differences after UV light exposure between the complementation groups XP-A, XP-C, XP-D, XP-E, XP-F, XP-G and an unaffected control. The results reveal that there is a graded change in gene expression patterns between the mildest, most similar to the control response (XP-E) and the severest form (XP-A) of the disease, with the exception of XP-D. Distinct differences between the complementation groups with neurological symptoms (XP-A, XP-D and XP-G) and without (XP-C, $X P-E$ and XP-F) were also identified. Therefore, this analysis has revealed distinct gene expression profiles for the XP complementation groups and the first step towards understanding the neurological symptoms of XP.
\end{abstract}

\section{Introduction}

The structural integrity of chromosomal DNA is of paramount importance for the survival of a cell, an organism and indeed an entire species [1]. The faithful passage of this nucleotide blueprint requires its stable transition between successive cellular generations [2]. Both replicating and non-replicating DNA are vulnerable to various forms of errors and lesions that constitute or lead to gene mutations [3]. To counteract the effects of mutagenic and carcinogenic agents all organisms are equipped with a sophisticated network of DNA repair systems that are essential for genetic stability [4]. The identification of several cancer syndromes in humans where the causative factor is a genetic mutation in a component of one or more of 
the DNA repair systems has highlighted the importance of maintaining DNA integrity.

Exogenous DNA mutagenesis is a consequence of external agents impacting on the integrity of cellular DNA. These agents can be divided into three classes: ultraviolet (UV) irradiation, ionising irradiation and alkylating agents. DNA repair is an important molecular defence system in which mutations caused by these agents in actively transcribed genes are preferentially repaired. All damaged DNA is repaired before DNA replication to prevent a mutation being transmitted to daughter cells [5]. DNA repair can occur by one of three cellular responses that involve direct reversal, excision or tolerance of the DNA damage.

UV irradiation primarily results in DNA damage in the form of 6,4 photoproducts and cyclobutane pyrimidine dimers which require excision from the surrounding DNA. Nucleotide excision repair (NER) involves an enzyme system that hydrolyses two phosphodiester bonds on either side of a lesion, creating an oligonucleotide surrounding the damage. NER has two distinct subpathways, global genomic repair (GGR) and transcription-coupled repair (TCR). GGR can operate at any location in the genome, and its efficiency varies depending on the type of lesion. In contrast, TCR specifically removes lesions that block RNA polymerases on the transcribed strands of active genes $[6,7]$ and eliminates different lesions at similar rates [8].

Since 1991 extensive research has been conducted on NER in humans. A wealth of information has become available since the cloning of the human repair genes, XPA to XPG and ERCC1 [9]. The discovery of defective NER in individuals with the rare heritable diseases xeroderma pigmentosum (XP), trichothiodystrophy (TTD) and Cockayne's syndrome (CS) and the subsequent isolation of UV-sensitive mutants of rodent cell lines led to the cloning of the human repair genes. Before XP was identified there was relatively little known about NER and DNA repair in general. As information regarding $X P$ is accumulated, more is being discovered about the NER proteins and their involvement in DNA repair and other processes. The role of UV-induced DNA damage in skin cancer and the importance of adequate repair systems to remove the damage are clearly illustrated in patients with XP $[10,11]$.

$\mathrm{XP}$ is a recessively inherited genetic disorder, which occurs at a frequency of 1:250,000 in the United States, but has a higher frequency in Japan and Mediterranean areas [12]. XP patients exhibit a 1000 times greater susceptibility to uniformly distributed melanomas, basal cell carcinomas and squamous cell carcinomas in sunlight-exposed areas of skin compared to unaffected individuals $[10,11,13]$. XP cells cannot efficiently carry out NER, and as a result there is an accumulation of errors as the damaged DNA tries to replicate itself [14].

$X P$ includes seven genetic subgroups known as complementation groups (XP-A to XP-G) that represent different genes in the NER pathway (Table 1). The functions of most of the XP and CS proteins associated with NER have been identified and mutations located in each of the genes have begun to be correlated with cellular functions and severity of the disease [15]. Currently, individuals are diagnosed with XP based on clinical criteria and identification of mutations in one of the NER genes. TTD and CS are different to XP as they do not have the higher rate of skin cancers and are characterized by growth and mental retardation. Mutation detection in these individuals is timeconsuming and costly as many require multiple genes to be screened before a mutation is identified. In addition to the high skin cancer incidence in XP, progressive neurological degeneration in the form of primary neuronal degeneration and mental retardation occurs in a significant number of patients, which is a result of mutations in specific XP genes.

Recent advances in biotechnology have seen the introduction of technologies that allow large numbers of genes and proteins to be analysed. This technology is a useful tool when studying complex disorders such as $\mathrm{XP}$, which are believed to involve changes in expression of many genes or proteins. One such example is microarrays that can detect expression levels of up to 30,000 genes at the same time. A number of studies have shown that gene expression profiling can identify sets of genes that allow identification of disease subtypes. For example, two distinct forms of diffuse large B-cell lymphoma were identified using gene profiling of 96 malignant and normal samples [16]. Similarly, Sorlie and colleagues [17] found that in a subset of patients, classification of breast cancer tumours by gene expression profiles could be used as a prognostic marker with respect to overall and relapse-free survival. Oestrogen receptor (ER) positive tumours could also be divided by gene expression profiles into two distinct subgroups with differing prognosis. The identification of distinct profiles for each XP complementation group would expedite the diagnostic process for new cases of $X P$ and further our understanding of some of the biological processes associated with NER deficiency. 
Table 1. Genes with brain-related function or expression that were significantly altered in association with neurological symptoms in XP

\begin{tabular}{|c|c|c|c|}
\hline Acc. No. & Name & Function & $P$ value \\
\hline AA464744 & KIAA 1111 & brain cDNA clone & 0.00004 \\
\hline R79082 & protein tyrosine phosphatase & cellular signalling pathways & 0.0004 \\
\hline N35888 & phosphomannomutase 2 (PMM2) & brain specific glycosylation factor & 0.0007 \\
\hline R93912 & glycogen synthase kinase 3 beta (GSK3 beta) & signal cascade of neuronal apoptosis & 0.0008 \\
\hline N70841 & GABA-B Receptor la & neurotransmitter receptor & 0.0008 \\
\hline T64482 & esterase D/formylglutathione hydrolase & expressed predominantly in brain & 0.0008 \\
\hline AA463251 & nucleosome assembly protein (NAP) & induces neuronal apoptosis & 0.0008 \\
\hline AA495858 & hydroxymethylbilane synthase & cell cycle and differentiation in glioma cells & 0.0023 \\
\hline AA460927 & translin & dendritic RNA binding comlex & 0.0034 \\
\hline $\mathrm{H} 10965$ & peroxisomal biogenesis factor 12 (PEX12) & $\begin{array}{l}\text { catalyses metabolism, peroxisome biogenesis disorder } \\
\text { (symptoms include neurodevelopmental delay) }\end{array}$ & 0.0041 \\
\hline N33331 & peroxisome proliferator activated receptor (PPAR) & anti-inflammatory and decreases free radical formation & 0.0062 \\
\hline AA456830 & diacylglycerol kinase (DAGK) & myelination & 0.0064 \\
\hline AA491225 & myotubular myopathy 1 (MTM1) & $\begin{array}{l}\text { expressed in brain, family members involved } \\
\text { in neuromuscular disorders }\end{array}$ & 0.0064 \\
\hline AA504333 & $\begin{array}{l}\text { endothelial-monocyte activating polypeptide II } \\
\text { (EMAPII) }\end{array}$ & brain inflammatory response & 0.0069 \\
\hline AA644088 & cathepsin C & upregulated after brain ischaemia & 0.0071 \\
\hline \multirow[t]{2}{*}{ H19522 } & exostoses-like protein 1 (EXTL1) & tumour suppressor, highly expressed in brain & 0.0076 \\
\hline & transducer of erbB2 (Tob) & tumour suppressor, highly expressed in brain & 0.0076 \\
\hline AA489331 & adenosine deaminase & metabolism of adenosine & 0.0083 \\
\hline
\end{tabular}

$\mathrm{XP}$ is a well characterized DNA repair disorder with expected outcomes of gene expression for the NER pathway after UV light irradiation. However, there is an unknown biological basis for neurological symptoms appearing in particular complementation groups of XP. Recent studies in neurological [18] and psychiatric disorders [19-21] have reported altered expression of genes with neurological expression and/or function in peripheral tissue, suggesting that identification of differential gene expression between complementation groups with the presence/absence of neurological symptoms in peripheral tissues such as skin fibroblasts may determine the genes or biological mechanisms involved in these previously unexplained clinical characteristics of XP.

In this study, 6000 gene cDNA microarrays were used to identify altered gene expression in control and XP fibroblast cell lines after UV light exposure. NER gene expression was altered in all of the XP cell lines compared to the control cell line, and distinct gene expression profiles for each XP complementation group were identified. Finally, genes involved in the neurological symptoms present in XPA, XPD and XPG complementation groups were also identified. This may shed some light on the biological cause of these previously unstudied symptoms of XP.

\section{Materials and methods}

\section{Control and XP fibroblast cell lines}

The control fibroblast cell line was obtained from the Molecular Genetics Laboratory, Hunter Area Pathology Service, John Hunter Hospital, Newcastle, Australia. The XP fibroblasts were obtained from the NIGMS Human Genetic Cell Repository, Coriell Institute for Medical Research. The following XP cell lines had 
known mutations: XPA (GM00544B) 2bp deletion A468-A469; XPC (GM00030A) 83bp insertion at 462; and XPD (GM00435) C to T substitution C2047T. XPE (GM01389A), XPG (GM3021B) and XPF (GM03542C) cell lines had a clinical history consistent with previously reported cases for these complementation groups. All the fibroblast cell lines were incubated with $1 \times$ DMEM (10\% FCS) at $37^{\circ} \mathrm{C}\left(5 \% \mathrm{CO}_{2}\right)$. To identify differential gene expression in the control and XP fibroblasts, each cell line was exposed to UV light to induce NER. A cell survival study, involving $2 \mathrm{~J} / \mathrm{m}^{2}$ UV light exposure over a time course followed by trypan blue exclusion to identify the percentage of cell survival, identified 1 minute of $2 \mathrm{~J} / \mathrm{m}^{2}$ UV light exposure sufficient to induce NER but not immediate cell death. Half of the control and XP cells were exposed to $2 \mathrm{~J} / \mathrm{m}^{2}$ UV light for $1 \mathrm{~min}$ in PBS when $100 \%$ confluent, and were allowed to recover for 1 hour.

\section{RNA extraction}

RNA was extracted from approximately $1 \times 10^{7}$ fibroblasts using TRlzol reagent as per manufacturer's instructions. Analysis and quantification of the RNA samples were performed by spectrophotometry (Cary 50 Bio UV-Visible spectrophotometer, Varian) using a ratio of $A_{260} / A_{280}$ as described previously [21]. RNA integrity was assessed by agarose/formaldehyde gels as described previously [21].

\section{Microarray procedure}

Total RNA $(25 \mu \mathrm{g})$ was used for indirect labelling of first-strand cDNA with Cy3 and Cy5 using the CyScribe cDNA Post-Labelling Kit (Amersham Pharmacia) as per manufacturer's instructions. The labelled cDNA was hybridized on ResGen 6K cDNA microarrays (Clive and Vera Ramaciotti Centre for Gene Function Analysis) and washed as per the CyScribe cDNA Post-Labelling Kit (Amersham Pharmacia) manufacturer's instructions. The microarrays were then scanned with an Axon 4000B microarray scanner and the image captured using GenePix Pro 3.0 software.

\section{Microarray data analysis}

The images were captured and analysed using GenePix Pro 3.0 and results tables containing fluorescence ratios were obtained. The control and XP fibroblast cell lines were all performed in duplicate with reverse labelling of the second microarray. All the reverse labelled microarray results were inverted to ensure all results were analysed as a ratio of treated/untreated. Areas or spots on the arrays that were affected by background were flagged and excluded from further analyses. GeneSpring 5.0 (Silicon genetics, USA) was used to normalize per spot and per array (Lowess curve) and to a $50^{\text {th }}$ percentile.

The relatedness of each XP complementation group and the control fibroblast cell line was tested by performing unsupervised cluster analysis using distance and correlation of all of the duplicate microarrays. The number of genes expressed in control and XP fibroblasts after UV light exposure was measured using a level of detection two-fold greater than the surrounding background. The number of genes in each cell line with greater than 1.5 fold alteration after UV light exposure was also measured. Statistically significant gene lists were created comparing the control fibroblasts and XP complementation groups using a non-parametric test (Wilcoxon-Mann-Whitney test) with a p-value cutoff of 0.05. Using these lists a dendrogram (experiment tree) was created using standard correlation to show the relationships between the expression profiles of the complementation groups. A second dendrogram (gene tree) was created to show relationships between the expression levels of genes across all the complementation groups. Percentage expected NER activity for each XP complementation group was then used to determine the accuracy of the clustering pattern. Significantly altered gene expression based on presence/absence of neurological symptoms was identified using the nonparametric test (Wilcoxon-Mann-Whitney test) with a $p$ value cutoff of 0.05 . Using this list a second dendrogram was created to show the relationships between the profiles of the XP complementation groups.

\section{Relative real-time PCR}

To confirm altered gene expression of STAT1 and LSD1 identified by microarray analysis CDNA relative semi-quantification was performed by real-time PCR as described previously [21].

\section{Results}

\section{Gene expression profiling}

The control fibroblasts expressed more genes than any of the XP fibroblast cell lines after UV light irradiation (Fig. 1), indicating that altered expression due to NER deficiency was being detected. Gene expression alterations greater than 1.5 fold, specific to each XP complementation group, were also identified (Fig. 2). Although there was greater expression in the control fibroblasts after UV light treatment, the number of genes altered by greater than 
1.5 fold was greater in some XP complementation groups, in particular XPD. Conversely, the XPA fibroblasts showed a low number of genes differentially expressed by greater than 1.5 fold (Fig. 2), which is reflective of the low number of genes expressed by the XPA fibroblast cultures after UV light exposure (Fig. 1).

To ascertain the relatedness of each XP complementation group and the control, standard correlation and distance were used to create a dendrogram to show relationships between gene expression profiles. Similarity of gene expression profiles is measured by distance on a dendrogram. Distance is measured by the vertical height of the branches of the dendrogram; therefore the closer the relatedness of one profile to another, the shorter the vertical branch. A second dendrogram (gene tree) was created using standard correlation and distance to show relationships between the expression levels of genes across the groups. Unsupervised clustering analysis was used to identify the relatedness of the gene expression profiles for the control fibroblasts and the XP complementation groups (Fig. 3). The relatedness of the control fibroblasts and XP complementation groups, with the exception of XPD, correlated directly with the expected

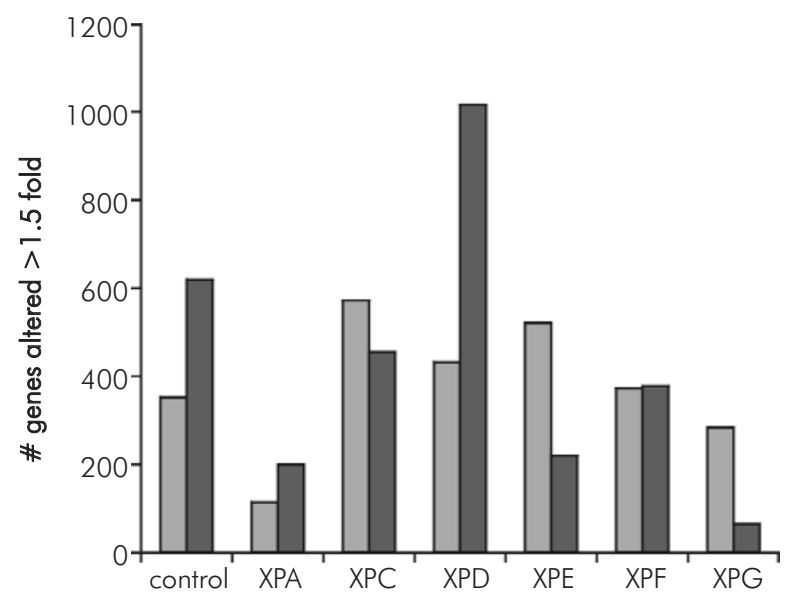

$$
\begin{aligned}
& \square \text { up-regulated } \\
& \square \text { down-regulated }
\end{aligned}
$$

Fig. 2. Number of genes altered greater than 1.5 fold in control and XP complementation group fibroblasts after $2 \mathrm{~J} / \mathrm{min} \mathrm{UV}$ light treatment. Control and XP fibroblasts cell line cultures were exposed to UV light. Numbers of differentially expressed genes were determined by identifying genes with a greater than 1.5 fold change in expression after UV light exposure. Similar numbers of genes were differentially expressed in the control and XP complementation groups with the exception of XPD which had a considerably higher number of genes down-regulated by greater than 1.5 fold and XPA which had a low number of differentially expressed genes, reflective of the low number of genes expressed in the XPA fibroblasts after UV light exposure percentage of NER activity present in each cell line. XPE has approximately $45 \%$ normal NER activity and was the most similar to the control profile as measured by distance on the dendrogram (i.e. there is vertical distance between all the other XP profiles and the branch joining control

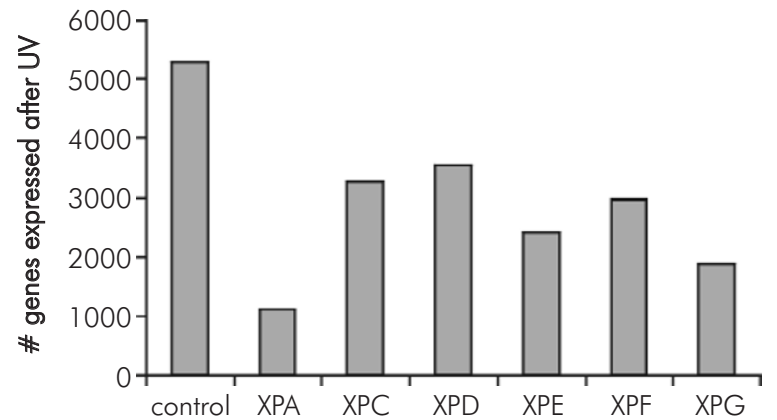

Fig. 1. Number of genes expressed in control and XP complementation group fibroblasts after $2 \mathrm{~J} / \mathrm{min}$ UV light treatment. Control and XP fibroblast cell line cultures were exposed to UV light. The number of genes expressed in each cell line was determined by the fluorescence of expressed genes being two-fold above surrounding background on the duplicate microarrays. A higher number of genes were expressed in the control fibroblasts after UV light exposure when compared to the XP complementation groups

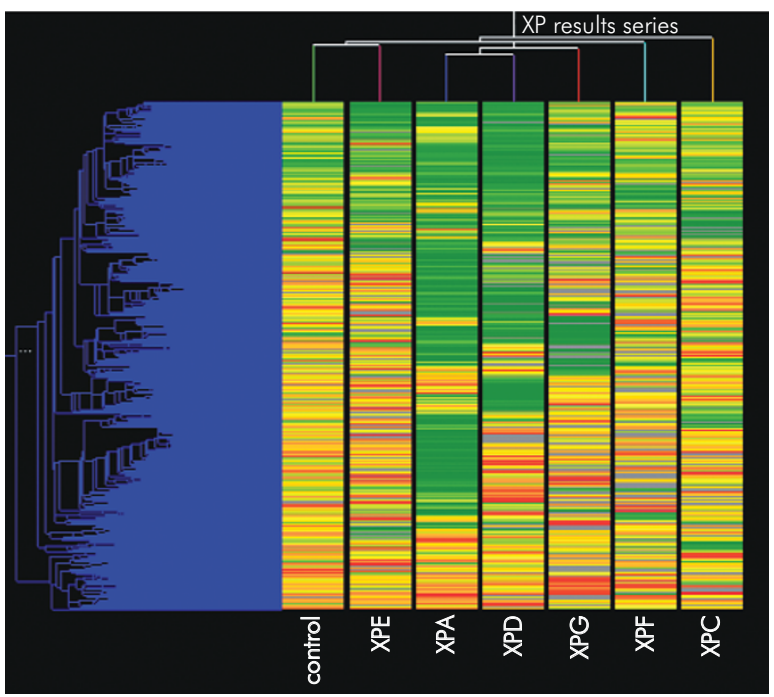

Fig. 3. Cluster analysis of control and XP complementation groups after UV light treatment. A comparison of control and XP complementation group with gene expression. Control and XP fibroblasts cell line cultures were exposed to UV light. Each column represents gene expression for each of the cell lines after UV light exposure. Downregulation is represented as green, up-regulation as red and normal expression as yellow. The dendrogram on top represents the relationship between and within the 7 cell lines. The dendrogram on the side represents relatedness of genes based on expression levels. The relatedness of the control fibroblasts and XP complementation groups with the exception of XPD correlated directly with the expected percentage of NER activity present in each cell line 
and XPE). XPF and XPC have approximately 20\% normal NER activity and were the next closest to the control profile respectively (represented as the next two shortest branches of the tree). XPG was closely related to XPF by distance and has approximately $10 \%$ normal NER activity. Finally XPA and XPD were closely correlated and the furthest in distance from the control profile. XPA has less than $2 \%$ of normal NER activity and XPD has approximately $30 \%$.

The unsupervised clustering showed that XPA, XPD and XPG profiles had a high correlation, suggesting that an alternative parameter to percentage of expected NER deficiency was influencing the profiles. To investigate the cause of the XPD profile not correlating with NER activity, and the presence of the XPA, XPD and XPG cluster, the presence/absence of neurological symptoms was used to identify significantly altered genes. 383 genes were significantly altered in the $X P$ groups with neurological symptoms (XPA, XPD, XPG) when compared to the XP groups without the symptoms (XPC, XPE, XPF). 18 highly altered $(P<0.01)$ genes have previously been reported to have brain-related function or expression (Table 1).

Cluster analysis of XP complementation groups exhibiting neurological symptoms using this list also identified distinct profiles associated with the symptoms (Fig. 4). The profiles for XPA, XPD and XPG (Fig. 4 red branches) were closely related as depicted by small joining branches, and distinctly different to control, XPC, XPE and XPF (Fig. 4 blue branches).

\section{Semi-quantitative real-time PCR}

Semi-quantitative real-time PCR using SybrGreen was used to confirm altered gene expression identified by microarray analysis. Initially, the expression of two housekeeping genes, 18s ribosomal RNA and $\beta$-Actin, were measured for each of the UV-treated and untreated control and XP fibroblasts cell lines. Two genes, signal transducer and activator of transcription 1 (STAT1) and lysine specific demethylase 1 (LSD1), having consistently altered expression across the cell lines from the microarray analysis, were chosen for confirmation. STAT1 and LSD1 exhibited similar fold change expression in the microarray and real-time PCR analysis (Table 2) after UV light treatment. LSD1 expression was not expressed at a detectable level in the control fibroblasts after UV light exposure. These results confirm that the microarray results were reflective of expression levels in the fibroblast cell lines before and after UV light treatment.

\section{Discussion}

Exposure to UV light initiates differential expression of a high numbers of genes in fibroblasts compared to, for example, cardiac myocytes [22]. The present study exemplifies the use of fibroblasts to study gene expression profiles in disorders with reduced NER activity as a high number of genes were expressed in the control fibroblasts after UV light exposure and a diminished number of genes were observed in the NER-deficient XP fibroblasts. The distinguishing feature of XP compared to other NER-deficient disorders is DNA repair or DNA replication deficiencies involving most of the genome [23]. For this reason, after UV light irradiation, it is to be expected that whole genome expression in XP fibroblasts will be considerably lower than in unaffected fibroblasts, due to the high levels of damaged DNA. This was confirmed in this study by the decrease in overall expression in the XP complementation groups compared

Table 2. Control and XP complementation groups microarray and relative real-time PCR fold change expression levels after UV light irradiation

\begin{tabular}{lcccc}
\hline Cell Line & \multicolumn{2}{c}{ LSD1 } & STAT1 \\
\cline { 2 - 5 } & $\begin{array}{c}\text { Microarray } \\
\text { Fold Change }\end{array}$ & $\begin{array}{c}\text { Real-Time PCR } \\
\text { Fold Change }\end{array}$ & $\begin{array}{c}\text { Microarray } \\
\text { Fold Change }\end{array}$ & $\begin{array}{c}\text { Real-Time PCR } \\
\text { Fold Change }\end{array}$ \\
\hline control & -4.6 & N/D & -1.9 & -5.4 \\
\hline XPA & -3.1 & -2.2 & -7.2 & -3.1 \\
\hline XPC & -5.8 & -2.2 & -1.8 & -5.2 \\
\hline XPD & -12.7 & -8.5 & -11.6 & -3.4 \\
\hline XPE & -5.0 & -4.5 & -2.6 & -4.6 \\
\hline$X P F$ & -2.8 & -2.8 & -2.2 & -9.3 \\
\hline$X P G$ & -5.5 & -1.0 & -1.5 & -3.6 \\
\hline N/D $=$ not detected & & & &
\end{tabular}


to the control after UV light treatment. The overall decrease in numbers of genes expressed was not reflected, however, in the number of genes altered in each XP complementation group compared to control. This result may be due to the NER deficiency resulting in altered expression of other DNA repair mechanisms, cell-cycle genes and apoptotic pathways.

Although all the XP complementation groups have some level of NER deficiency, they have distinct characteristics such as differing levels of UV light sensitivity, skin cancer susceptibility and neurological symptoms. Distinct gene expression profiles were identified for each complementation group, with percentage NER activity showing a relationship to relatedness of profiles. The XPE profile was the most similar to the control profile, which is to be expected as XPE has relatively high levels of NER activity and the least severe symptoms [24]. The remaining XP complementation groups followed the pattern of the lower the percentage NER activity the further the profile from control, with the exception of XPD. XPD showed a similar profile to XPA, which has a $<2 \%$ NER activity, and was equal furthest from control.

The presence of neurological symptoms in XPA, XPB, XPD and XPG has rarely been investigated. In this study, a list of genes significantly different in XPA, XPD and XPG compared to control, XPC, XPE and XPF was generated and cluster analysis performed to identify distinct gene expression profiles related to the presence of neurological symptoms. Many of the altered genes have neurological functions and have previously been reported to be expressed in brain tissue and, although not one of the aims of this study, further analysis of the data may identify underlying causes for the symptoms. This current study suggests that deficiencies in NER probably result in the accumulation of DNA damage in neurological tissue, probably as a result of endogenous insult.

Finally, relative real-time PCR was used to confirm the altered expression of two genes in the control and XP cell lines. STAT1 and LSD1 showed similar expression between the microarrays and real-time PCR, suggesting that the microarray data is semi-quantitative, and more importantly is identifying altered regulation of genes in each sample tested.

In conclusion, the data herein suggests that cluster analysis can identify distinct gene expression patterns between XP complementation groups. Relative realtime PCR confirmation has also shown that the data are representative of gene expression in the control and XP cell lines before and after UV irradiation. The

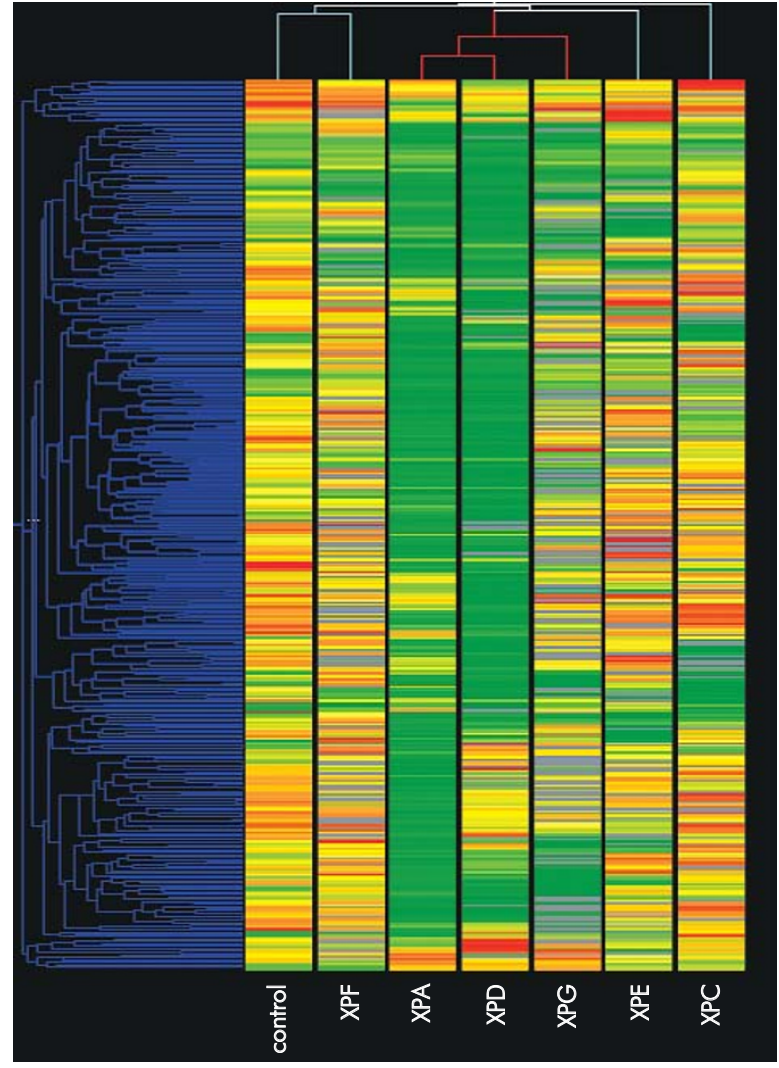

Fig. 4. Cluster analysis of neurological symptoms present in control and XP complementation groups. A comparison of control and XP complementation group with presence/absence of neurological symptoms and gene expression. Control and XP fibroblasts cell line cultures were exposed to UV light. Each column represents expression in each of the cell lines of 383 genes significantly differentially expressed in XP complementation groups with and without neurological symptoms. Down-regulation is represented as green, upregulation as red and normal expression as yellow. The dendrogram on top represents the relationship between and within the two groups; blue branches represent absence and red branches represent presence of neurological symptoms. The dendrogram on the side represents relatedness of genes based on expression levels. A cluster of red branches is clearly depicted in the top dendrogram indicating a distinct gene expression profile for the presence of neurological symptoms in $\mathrm{XP}$

identification of distinct gene expression profiles for XP complementation groups will assist in diagnosis of the disease as well as further our understanding of the biology of XP. In addition, the understanding of the biological cause of neurological symptoms in XP will be furthered with the instigation of gene expression profiling in XP complementation groups.

\section{Acknowledgments}

The authors would like to thank the Hunter Medical Research Institute and the NBN Children's Cancer group for their support of this project. 


\section{References}

1. Hendrickson EA. Insights from model systems: cell-cycle regulation of mammalian DNA double-strand-break repairs. Am J Hum Genet 1997; 61:795-800.

2. Singer M and Berg P. Genes and genomes: a changing perspective. USA: University Science Press 1991.

3. Klug WS and Cummings MR. Concepts in Genetics. USA: Macmillan College Publishing Company Inc. 1994.

4. Weeda GA. A mutation in the XPB/ERCC3 DNA reapir transcription gene associated with trichothiodystrophy. Am J Hum Genet 1997; 60: 320-329.

5. Woods CG. DNA repair disorders. Arch Dis Child 1998; 78: 178-184.

6. Bohr VA, Smith CA, Okumoto DS and Hanawalt PC. DNA repair in an active gene: removal of pyrimidine dimers from the DHFR gene of $\mathrm{CHO}$ cells is much more efficient than in the genome overall. Cell 1985; 40: 359-369.

7. Mellon I, Spivak G and Hanawalt PC. Selective removal of transcription-blocking DNA damage from the transcribed strand of mammalian DHFR gene. Cell 1987; 51: 241-249.

8. van Hoffen A, Venema J, Meschini R, van Zeeland AA and Mullenders $L$. Transcription-coupled repair removes both cyclobutane pyrimidine dimers and 6-4 photoproducts with equal efficiency and in a sequential way from transcribed DNA in xeroderma pigmentosum group C fibroblasts. EMBO J 1995; 14: 360-367.

9. Legerski R and Peterson C. Expression cloning of a human DNA repair gene involved in xeroderma pigmentosum group $\mathrm{C}$. Nature 1992; 359: 70-73.

10. Bootsma D, Kraemer KH, Cleaver JE and Hoeijmakers JHJ. Nucleotide excision repair syndromes: xeroderma pigmentosum and trichothiodystrophy. In: Vogelstein B, Kinzler KW, editors. The genetic basis of human cancer: McGraw-Hill 1998; 245-274.

11. Friedberg EC, Walker GC and Siede W. DNA repair and mutagenesis. Washington: ASM Press 1995.

12. Cleaver JE, Kraemer KH. Xeroderma Pigmentosum and Cockayne's Syndrome. In: The meetabolic and molecular basis of inherited disease. New York: McGraw-Hill 1995; 4393-4419.

13. Kraemer KH. Sunlight and skin cancer: another link revealed. Proc Natl Acad Sci U S A 1997; 94: 11-14.

14. Wood RD. Nucleotide excision repair in mammalian cells. J Biol Chem 1997; 272: 23465-23468.

15. Thompson LH. DNA repair in mammalian cells. Totowa, NJ: Humana Press 1998.

16. Alizadeh AA, Eisen MB, Davis RE, Ma C, Lossos IS, Rosenwald A, Boldrick JC, Sabet H, Tran T, Yu X, Powell JI, Yang L, Marti GE, Moore T, Hudson J Jr, Lu L, Lewis DB, Tibshirani R, Sherlock G, Chan WC, Greiner TC, Weisenburger DD, Armitage JO, Warnke R, Levy R, Wilson W, Grever MR, Byrd JC, Botstein D, Brown PO and Staudt LM. Distinct types of diffuse large B-cell lymphoma identified by gene expression profiling. Nature 2000 ; 403: 503-511.

17. Sorlie T, Perou CM, Tibshirani R, Aas T, Geisler S, Johnsen $H$, Hastie T, Eisen MB, van de Rijn M, Jeffrey SS, Thorsen T, Quist $H$, Matese JC, Brown PO, Botstein D, Eystein Lonning P and Borresen-Dale AL. Gene expression patterns of breast carcinomas distinguish tumor subclasses with clinical implications. Proc Natl Acad Sci U S A 2001; 98: 10869-10874

18. Kalman J, Kitaika K, Pakaski M, Zvara A, Juhasz A, Vincze G, Janka $Z$ and Puskas LG. Gene expression profile analysis of lymphocytes from Alzheimer's patients. Psychiatr Genet 2005; 15: 1-6.

19. Tsuang MT, Nossova N, Yager T, Tsuang MM, Guo SC, Shyu KG, Glatt SJ and Liew CC. Assessing the validity of blood-based gene expression profiles for the classification of schizophrenia and bipolar disorder: a preliminary report. Am J Med Genet B Neuropsychiatr Genet 2005; 133: 1-5.

20. Vawter MP, Ferran E, Galke B, Cooper K, Bunney WE and Byerley W. Microarray screening of lymphocyte gene expression differences in a mutiplex schizophrenia pedigree. Schizophr Res 2004; 67: 41-52.

21. Bowden NA, Weidenhofer J, Scott RJ, Schall U, Todd J, Michie PT and Tooney PA. Preliminary investigation of gene expression profiles in peripheral blood lymphocytes in schizophrenia. Schizophr Res 2006; 82: 175-183.

22. Boerma M, van der Wees CG, Vrieling $H$, Svensson JP, Wondergem J, van der Laarse A, Mullenders LH and van Zeeland AA. Microarray analysis of gene expression profiles of cardiac myocytes and fibroblasts after mechanical stress, ionising or ultraviolet radiation. BMC Genomics 2005; 6: 6.

23. Cleaver JE. Cancer in xeroderma pigmentosum and related disorders if DNA repair. Nat Rev Cancer 2005; 5: 564-573.

24. van Steeg $\mathrm{H}$ and Kraemer KH. Xeroderma pigmentosum and the role of UV-induced DNA damage in skin cancer. Mol Med Today 1999; 5: 86-94. 